PROCEEDINGS OF THE

AMERICAN MATHEMATICAL SOCIETY

Volume 138, Number 6, June 2010, Pages 2169-2180

S 0002-9939(10)10230-5

Article electronically published on January 14, 2010

\title{
STABILITY IN DISTRIBUTION OF MILD SOLUTIONS TO STOCHASTIC PARTIAL DIFFERENTIAL EQUATIONS
}

\author{
JIANHAI BAO, ZHENTING HOU, AND CHENGGUI YUAN
}

(Communicated by Richard C. Bradley)

\begin{abstract}
In the present paper, we investigate stochastic partial differential equations. By introducing a suitable metric between the transition probability functions of mild solutions, we derive sufficient conditions for stability in distribution of mild solutions. Consequently, we generalize some existing results to infinite dimensional cases. Finally, one example is constructed to demonstrate the applicability of our theory.
\end{abstract}

\section{INTRODUCTION}

Recently stochastic partial differential equations in a separable Hilbert space have been studied by many authors and various results on the existence, uniqueness, stability, invariant measures, and other quantitative and qualitative properties of solutions have been established. For example, in their books [6, 7], Da Prato and Zabczyk established systematic theory of the existence and uniqueness and the ergodicity for infinite dimensional systems. Moreover the theory of stability has attracted a great deal of attention. For example, the almost sure stability and the mean square stability were considered in Caraballo and Real [2, Caraballo and Liu [3], Chow [5], Govindan [8], Haussmann [9], Ichikawa [10, 11, Liu and Mandrekar [12, and Taniguchi [13, to name a few.

However, in many practical situations, almost sure stability and mean square stability are sometimes too strong. Therefore, we want to know whether or not the probability distribution of the mild solutions will converge weakly to some distribution (but not necessarily to zero). Such convergence is called the stability in distribution and the limit distribution is known as a stationary distribution. For the finite dimensional case, Basak et al. 1 considered such stability for semi-linear stochastic differential equations with Markovian switching. Then, Yuan et al. [14] extended by Lyapunov function approaches the results [1] to cover a class of much more general stochastic differential equations with Markovian switching. Since the mild solutions do not have stochastic differential, a significant consequence of this fact is that we cannot employ the Itô formula for mild solutions directly in most of our arguments. Therefore, it is not possible to investigate directly by the approaches

Received by the editors March 16, 2009, and, in revised form, September 23, 2009, and September 24, 2009.

2010 Mathematics Subject Classification. Primary 60H15, 60H30.

Key words and phrases. Stochastic partial differential equation, mild solution, stability in distribution.

This work was partially supported by the NNSF of China (Grant No. 10671212). 
of Yuan et al. [14] the stability in distribution of mild solutions to stochastic partial differential equations. We would like to point out that the tightness of the mild solutions together with the asymptotic closeness of mild solutions starting at two different initial data would give stability in distribution. Since ultimate boundedness in mean square implies tightness, the method used in Lemma 3.1, which discusses the tightness of solutions, is similar to that of [12], where the case of exponentially ultimate boundedness in mean square is investigated; while Lemma 3.2 . which studies the asymptotic closeness of mild solutions starting at two different initial data, is new for stochastic partial differential equations. Moreover, the key contribution of our work is, by introducing from Ichikawa [10] an approximating system and constructing an appropriate metric between transition probability functions of mild solutions, to give sufficient conditions for stability in distribution of mild solutions. To the best of our knowledge, to date there are few papers which are concerned with the stability in distribution of mild solutions to stochastic partial differential equations, so we want to close the gap.

The format of the rest of this paper is organized as follows. In section 2, we collect basic definitions and recall some preliminary results. In section 3 , several lemmas which lay the foundation for stability analysis are presented and the main result is derived by constructing a suitable metric between transition probability functions of mild solutions. In order to demonstrate the applicability of our theory, one example is established in the last section.

\section{Preliminaries}

Let $\left\{\Omega, \mathcal{F},\left\{\mathcal{F}_{t}\right\}_{t \geq 0}, \mathcal{P}\right\}$ be a complete probability space equipped with some filtration $\left\{\mathcal{F}_{t}\right\}_{t \geq 0}$ satisfying the usual conditions (i.e., it is right continuous and $\mathcal{F}_{0}$ contains all $\mathcal{P}$-null sets). Let $H, K$ be two real separable Hilbert spaces and denote their inner products by $\langle\cdot, \cdot\rangle_{H},\langle\cdot, \cdot\rangle_{K}$ and their norms by $\|\cdot\|_{H},\|\cdot\|_{K}$, respectively. We denote by $\mathcal{L}(K, H)$ the set of all linear bounded operators from $K$ into $H$, equipped with the usual operator norm $\|\cdot\|$. Throughout this paper, we always use the symbol $\|\cdot\|$ to denote norms of operators regardless of the spaces potentially involved when no confusion can possibly arise.

Denote by $\{W(t), t \geq 0\}$ a $K$-valued $\left\{\mathcal{F}_{t}\right\}_{t \geq 0}$-Wiener process defined on the probability space $\left\{\Omega, \mathcal{F},\left\{\mathcal{F}_{t}\right\}_{t \geq 0}, \mathcal{P}\right\}$ with covariance operator $Q$, i.e.,

$$
E\langle W(t), x\rangle_{K}\langle W(s), y\rangle_{K}=(t \wedge s)\langle Q x, y\rangle_{K}, \quad \forall x, y \in K
$$

where $Q$ is a positive, self-adjoint, trace class operator on $K$. In particular, we call such $\{W(t), t \geq 0\}$ a $K$-valued $Q$-Wiener process with respect to $\left\{\mathcal{F}_{t}\right\}_{t \geq 0}$. According to Da Prato et al. [6, Proposition 4.1, p. 87], $W(t)$ is defined by

$$
W(t)=\sum_{n=1}^{\infty} \sqrt{\lambda_{n}} \beta_{n}(t) e_{n}, \quad t \geq 0
$$

where $\beta_{n}(t)(n=1,2,3, \cdots)$ is a sequence of real valued standard Brownian motions mutually independent on the probability space $\left\{\Omega, \mathcal{F},\left\{\mathcal{F}_{t}\right\}_{t \geq 0}, \mathcal{P}\right\},\left(\lambda_{n}, n \in N\right)$ are the eigenvalues of $Q$ and $\left(e_{n}, n \in N\right)$ are the corresponding eigenvectors. That is,

$$
Q e_{n}=\lambda_{n} e_{n}, \quad n=1,2,3, \cdots
$$


To define stochastic integrals with respect to the $Q$-Wiener process $W(t)$, we introduce the subspace $K_{0}=Q^{\frac{1}{2}}(K)$ of $K$ which, endowed with the inner product

$$
\langle u, v\rangle_{K_{0}}=\left\langle Q^{-\frac{1}{2}} u, Q^{-\frac{1}{2}} v\right\rangle_{K},
$$

is a Hilbert space. Let $\mathcal{L}_{2}^{0}=\mathcal{L}_{2}\left(K_{0}, H\right)$ denote the space of all Hilbert-Schmidt operators from $K_{0}$ into $H$. It turns out to be a separable Hilbert space equipped with the norm

$$
\|\Phi\|_{\mathcal{L}_{2}^{0}}^{2}=\operatorname{tr}\left(\left(\Phi Q^{\frac{1}{2}}\right)\left(\Phi Q^{\frac{1}{2}}\right)^{*}\right) \quad \text { for any } \Phi \in \mathcal{L}_{2}^{0} .
$$

Clearly, for any bounded operators $\Phi \in \mathcal{L}(K, H)$, this norm reduces to $\|\Phi\|_{\mathcal{L}_{2}^{0}}^{2}=$ $\operatorname{tr}\left(\Phi Q \Phi^{*}\right)$. Let $\Phi:(0, \infty) \rightarrow \mathcal{L}_{2}^{0}$ be a predictable, $\mathcal{F}_{t^{-}}$adapted process such that for any $t \geq 0$

$$
\int_{0}^{t} E\|\Phi(s)\|_{\mathcal{L}_{2}^{0}}^{2} d s<\infty .
$$

Then, we can define an $H$-valued stochastic integral

$$
\int_{0}^{t} \Phi(s) d W(s)
$$

which is a continuous square integrable martingale. For that construction, refer to Da Prato et al. 6, pp. 90-96].

In this paper, we consider the semi-linear stochastic partial differential equation

$$
d X(t)=[A X(t)+F(X(t))] d t+G(X(t)) d W(t), \quad t \geq 0,
$$

with initial datum $X(0)=\xi \in H$. Throughout this paper, for the existence and uniqueness of the mild solutions, we shall impose the following assumptions:

(H1) $A$, generally unbounded, is the infinitesimal generator of a $C_{0}$-semigroup $T(t), t \geq 0$, of contraction.

(H2) The mappings $F: H \rightarrow H, G: H \rightarrow \mathcal{L}(K, H)$ are both Borel measurable and satisfy the Lipschitz condition

$$
\|F(x)-F(y)\|_{H}+\|G(x)-G(y)\|_{\mathcal{L}_{2}^{0}} \leq L\|x-y\|_{H},
$$

for some constant $L>0$ and arbitrary $x, y \in H$.

For convenience, we recall from Ichikawa [10] two kinds of solutions to (2.1).

Definition 2.1. A stochastic process $\{X(t), t \in[0, T]\}, 0 \leq T<\infty$, is called a strong solution of (2.1) if

(i) $X(t)$ is adapted to $\mathcal{F}_{t}$ and continuous in $t$ with probability 1 ;

(ii) $X(t) \in \mathcal{D}(A)$, the domain of $A$, on $[0, T] \times \Omega$ with $\int_{0}^{T}\|A X(t)\|_{H} d t<\infty$ with probability one,

$$
X(t)=\xi+\int_{0}^{t}[A X(s)+F(X(s))] d s+\int_{0}^{t} G(X(s)) d W(s)
$$

for all $t \in[0, T]$ with probability one.

In general, this concept is rather strong, and a weaker one described below is more appropriate for practical purposes. 
Definition 2.2. A stochastic process $\{X(t), t \in[0, T]\}, 0 \leq T<\infty$, is called a mild solution of (2.1) if

(i) $X(t)$ is adapted to $\mathcal{F}_{t}$;

(ii) $X(t)$ is measurable and $\int_{0}^{T}\|X(t)\|_{H}^{2} d s<\infty$ with probability 1 ,

$$
X(t)=T(t) \xi+\int_{0}^{t} T(t-s) F(X(s)) d s+\int_{0}^{t} T(t-s) G(X(s)) d W(s)
$$

for all $t \in[0, T]$ with probability one.

Remark 2.1. Note from [10, Proposition 2.1] that if $X(t), t \in[0, T]$, is a strong solution of (2.1), then it is also a mild solution.

Under the conditions $(H 1)$ and $(H 2)$, we have the following existence-anduniqueness result of mild solutions, which is stated as a lemma.

Lemma 2.1 ([10, Theorem 2.1]). Let $\xi \in H$ be $\mathcal{F}_{0}$ measurable with $E\|\xi\|^{p}<\infty$ for some integer $p \geq 2$. Then, under the conditions (H1) and (H2), there exists a unique mild solution $X^{\xi}(t)$ or simply $X(t)$ to (2.1) in $C\left(0, T ; L^{p}(\Omega, \mathcal{F}, P ; H)\right)$.

Remark 2.2. Under the conditions $(H 1)$ and $(H 2)$, the mild solution $X(t), t \in[0, T]$, to (2.1) is a strong Markov process [6. Theorem 9.15, p. 256].

Let $p(t, \xi, d \zeta)$ denote the transition probability of the process $y(t)$ with the initial state $y(0)=\xi$. Denote by $P(t, \xi, \Gamma)$ the probability of event $\{y(t) \in \Gamma\}$ given initial condition $y(0)=\xi$, i.e.

$$
P(t, \xi, \Gamma)=\int_{\Gamma} p(t, \xi, d \zeta)
$$

with $\Gamma \in \mathscr{B}(H)$ which denotes the Borel $\sigma$-field of $H$.

Since we are mainly interested in the stability in distribution of the mild solution $X(t)$ to (2.1), we introduce the notion of such stability.

Definition 2.3. The process $y(t)$ with the initial state $y(0)=\xi$ is said to be stable in distribution if there exists a probability measure $\pi(\cdot)$ on $H$ such that the transition probability $p(t, \xi, d y)$ of $y(t)$ converges weakly to $\pi(d y)$ as $t \rightarrow \infty$ for any $\xi \in H$. (2.1) is said to be stable in distribution if $X(t)$ is stable in distribution.

Remark 2.3. Since the mild solution $X(t)$ to (2.1) is a strong Markov process, using the Kolmogorov-Chapman equation, it is not difficult to show that the stability in distribution of mild solution $X(t)$ implies the existence of a unique invariant probability measure for mild solution $X(t)$.

For our purpose, we introduce the Itô formula, which will play an important role for our later stability analysis.

Let $R$ be a real number, $R_{+}$be a non-negative real number, and $C^{2}(H ; R+)$ denote the space of all real valued non-negative functions $V$ on $H$ with properties:

(i) $V(x)$ is twice (Fréchet) differentiable in $x$;

(ii) $V_{x}(x)$ and $V_{x x}(x)$ are both continuous in $H$ and $\mathcal{L}(H)=\mathcal{L}(H, H)$, respectively.

Lemma 2.2 ([10, Corollary 1.2]). Suppose $V \in C^{2}(H ; R+)$ and $\{X(t), t \geq 0\}$ is a strong solution to (2.1); for $t \geq 0$

$$
V(X(t))=V(\xi)+\int_{0}^{t} \mathcal{L} V(X(s)) d s+\int_{0}^{t}\left\langle V_{x}(X(s)), G(X(s)) d W(s)\right\rangle_{H},
$$


where, $\forall x \in \mathcal{D}(A)$,

$$
\mathcal{L} V(x)=\left\langle V_{x}(x), A x+F(x)\right\rangle_{H}+\frac{1}{2} \operatorname{tr}\left(V_{x x}(x) G(x) Q G^{*}(x)\right) .
$$

Since the mild solutions do not have stochastic differential, a significant consequence of this fact is that we cannot employ the Itô formula for mild solutions directly in most of our arguments. Following from Ichikawa [10, we introduce the approximating system of (2.1), for $t \geq 0$,

$$
\begin{aligned}
d X_{n}(t) & =A X_{n}(t) d t+R(n) F\left(X_{n}(t)\right) d t+R(n) G\left(X_{n}(t)\right) d W(t), \\
X(0) & =R(n) \xi \in \mathcal{D}(A),
\end{aligned}
$$

where $n \in \rho(A)$, the resolvent set of $A$, and $R(n)=n R(n, A), R(n, A)$ is the resolvent of $A$. Similar to operator $\mathcal{L}$ defined in Lemma 2.2. the operator $\mathcal{L}_{n}$ associated with (2.2), for any $x \in \mathcal{D}(A)$, can be defined by

$$
\mathcal{L}_{n} V(x)=\left\langle V_{x}(x), A x+R(n) F(x)\right\rangle_{H}+\frac{1}{2} \operatorname{tr}\left[V_{x x}(x) R(n) G(x) Q(R(n) G(x))^{*}\right] .
$$

Lemma 2.3 ([10, Lemma 3.1]). Under conditions $(H 1)$ and $(H 2)$, (2.2) has a unique strong solution $X_{n}(t)$ which lies in $C\left(0, T ; L^{2}(\Omega, \mathcal{F}, P ; H)\right.$ ) for all $T \geq 0$. Moreover, $X_{n}(t)$ converges to the mild solution $X(t)$ to (2.1) in $C\left(0, T ; L^{2}(\Omega, \mathcal{F}, P ; H)\right)$ as $n \rightarrow \infty$.

\section{Stability IN Distribution OF THE MiLD SOlUtion}

In this section, we begin to consider the stability in distribution of the mild solution $X(t)$ of (2.1). Since the proof of the main result is rather technical, we prepare several lemmas below.

Lemma 3.1. Let conditions $(H 1)$ and $(H 2)$ hold. Assume that there exists a function $V(x) \in C^{2}(H ; R+)$ such that for any $x \in H$,

$$
\begin{aligned}
c\|x\|_{H}^{2} & \geq V(x)+\|x\|_{H}\left\|V_{x}(x)\right\|_{H}+\|x\|_{H}^{2}\left\|V_{x x}(x)\right\|, \\
c_{1}\|x\|_{H}^{2} & \leq V(x),
\end{aligned}
$$

where $c$ and $c_{1}$ are certain positive constants. Moreover, assume that there are constants $\lambda_{1}>0$ and $\beta \in R_{+}$satisfying

$$
\mathcal{L} V(x) \leq-\lambda_{1} V(x)+\beta, \quad x \in \mathcal{D}(A) .
$$

Then, for any $\xi \in H$ and $\epsilon>0$, there exists a constant $M>0$ such that for any $t \geq 0$,

$$
P\left\{\|X(t)\|_{H} \geq M\right\}<\epsilon .
$$

Proof. The method used here is similar to that of [12, Theorem 2.1]; we shall give a detailed proof to keep the paper self-contained. Applying the Itô formula 
(i.e. Lemma 2.2) to the function $V(t, x)=e^{\lambda_{1} t} V(x)$ and the strong solution $X_{n}(t)$ to (2.2), for any $t \geq 0$,

$$
\begin{aligned}
\mathbb{E}\left(e^{\lambda_{1} t} V\left(X_{n}(t)\right)\right) & =\mathbb{E} V(R(n) \xi)+\mathbb{E} \int_{0}^{t} e^{\lambda_{1} s}\left[\lambda_{1} V\left(X_{n}(s)\right)+\mathcal{L}_{n} V\left(X_{n}(s)\right)\right] d s \\
& =\mathbb{E} \int_{0}^{t} e^{\lambda_{1} s}\left[\lambda_{1} V\left(X_{n}(s)\right)+\mathcal{L} V\left(X_{n}(s)\right)\right] d s \\
& +\mathbb{E} \int_{0}^{t} e^{\lambda_{1} s}\left\langle V_{x}\left(X_{n}(s)\right),(R(n)-I) F\left(X_{n}(s)\right)\right\rangle_{H} d s \\
& +\frac{1}{2} \mathbb{E} \int_{0}^{t} e^{\lambda_{1} s} \operatorname{tr}\left[V_{x x}\left(X_{n}(s)\right) R(n) G\left(X_{n}(s)\right) Q\left(R(n) G\left(X_{n}(s)\right)\right)^{*}\right] d s \\
& -\frac{1}{2} \mathbb{E} \int_{0}^{t} e^{\lambda_{1} s} \operatorname{tr}\left[V_{x x}\left(X_{n}(s)\right) G\left(X_{n}(s)\right) Q G^{*}\left(X_{n}(s)\right)\right] d s .
\end{aligned}
$$

By (3.2), it therefore follows that

$$
\begin{aligned}
\mathbb{E}\left(e^{\lambda_{1} t} V\left(X_{n}(t)\right)\right) & \leq \mathbb{E} V(R(n) \xi)+\frac{\beta}{\lambda_{1}}\left(e^{\lambda_{1} t}-1\right) \\
& +\mathbb{E} \int_{0}^{t} e^{\lambda_{1} s}\left\langle V_{x}\left(X_{n}(s)\right),(R(n)-I) F\left(X_{n}(s)\right)\right\rangle_{H} d s \\
& +\frac{1}{2} \mathbb{E} \int_{0}^{t} e^{\lambda_{1} s} \operatorname{tr}\left[V_{x x}\left(X_{n}(s)\right) R(n) G\left(X_{n}(s)\right) Q\left(R(n) G\left(X_{n}(s)\right)\right)^{*}\right] d s \\
& -\frac{1}{2} \mathbb{E} \int_{0}^{t} e^{\lambda_{1} s} \operatorname{tr}\left[V_{x x}\left(X_{n}(s)\right) G\left(X_{n}(s)\right) Q G^{*}\left(X_{n}(s)\right)\right] d s
\end{aligned}
$$

On the other hand, taking into account Lemma 2.3 and combining the dominated convergence theorem yield that

$$
\mathbb{E}\left(e^{\lambda_{1} t} V(X(t))\right) \leq \mathbb{E} V(\xi)+\frac{\beta}{\lambda_{1}}\left(e^{\lambda_{1} t}-1\right) .
$$

This, together with (3.1), gives that

$$
\mathbb{E}\|X(t)\|_{H}^{2} \leq \frac{\lambda_{1} \mathbb{E} V(\xi)+\beta}{c_{1} \lambda_{1}} .
$$

Now, for any $M>0$, by the Chebyshev inequality

$$
P\left\{\|X(t)\|_{H} \geq M\right\} \leq \frac{\mathbb{E}\|X(t)\|_{H}^{2}}{M^{2}} .
$$

Then, the required assertion (3.3) follows immediately from (3.4).

Remark 3.1. Under the conditions of Lemma 3.1, it is easy to observe by (3.3) that the family $\{p(t, \xi, d \zeta): t \geq 0\}$ is tight. That is, for any $\varepsilon>0$ there is a compact subset $\mathcal{K}=\mathcal{K}(\xi, \varepsilon)$ of $H$ such that

$$
P(t, \xi, \mathcal{K}) \geq 1-\varepsilon
$$


In what follows we need to consider the difference between two mild solutions of (2.1) starting from different initial datums; namely, for any $t \geq 0$,

$$
\begin{aligned}
X^{\xi}(t)-X^{\eta}(t) & =T(t) \xi-T(t) \eta+\int_{0}^{t} T(t-s)\left[F\left(X^{\xi}(s)\right)-F\left(X^{\eta}(s)\right)\right] d s \\
& +\int_{0}^{t} T(t-s)\left[G\left(X^{\xi}(s)\right)-G\left(X^{\eta}(s)\right)\right] d W(s) .
\end{aligned}
$$

Now, for $t \geq 0$ we introduce an approximating system in correspondence with (3.5):

$$
\begin{aligned}
d\left[X_{n}^{\xi}(t)-X_{n}^{\eta}(t)\right] & =A\left[X_{n}^{\xi}(t)-X_{n}^{\eta}(t)\right] d t+R(n)\left[F\left(X_{n}^{\xi}(t)\right)-F\left(X_{n}^{\eta}(t)\right)\right] d t \\
& +R(n)\left[G\left(X_{n}^{\xi}(t)\right)-G\left(X_{n}^{\eta}(t)\right)\right] d W(t), \\
X_{n}^{\xi}(0)-X_{n}^{\eta}(0) & =R(n)(\xi-\eta) \in \mathcal{D}(A),
\end{aligned}
$$

where $n \in \rho(A)$, the resolvent set of $A$, and $R(n)=n R(n, A), R(n, A)$ is the resolvent of $A$. For given $U \in C^{2}(H ; R+)$, define an operator $\mathcal{L}_{n} U: H \times H \rightarrow R$ associated with (3.6) for any $x, y \in \mathcal{D}(A)$ by

$$
\begin{aligned}
\mathcal{L}_{n} U(x, y) & =\left\langle U_{x}(x-y), A(x-y)+R(n)(F(x)-F(y))\right\rangle_{H} \\
& +\frac{1}{2} \operatorname{tr}\left(U_{x x}(x-y) R(n)(G(x)-G(y)) Q(R(n)(G(x)-G(y)))^{*}\right) .
\end{aligned}
$$

Lemma 3.2. Let conditions $(H 1)$ and $(H 2)$ hold. For any $x \in H$ assume that there exists a function $U(x) \in C^{2}(H ; R+)$ such that with some constants $d, c_{2} \geq 0$,

$$
\begin{aligned}
d\|x\|_{H}^{2} & \geq U(x)+\|x\|_{H}\left\|U_{x}(x)\right\|_{H}+\|x\|_{H}^{2}\left\|U_{x x}(x)\right\|, \\
c_{2}\|x\|_{H}^{2} & \leq U(x) .
\end{aligned}
$$

Assume moreover that there exists a constant $\lambda_{2}>0$ such that for any $x, y \in \mathcal{D}(A)$

$$
\mathcal{L} U(x, y) \leq-\lambda_{2} U(x-y)
$$

Then, for any $\epsilon>0$ and any compact subset $\mathcal{K}$ of $H$, there exists a $T=T(\epsilon, \mathcal{K})>0$ such that

$$
P\left\{\left\|X^{\xi}(t)-X^{\eta}(t)\right\|_{H}<\epsilon\right\} \geq 1-\epsilon, \quad t \geq T,
$$

whenever $\xi, \eta \in \mathcal{K}$.

Proof. It is easy to see from (3.7) that $U(0)=0$. For any $\epsilon \in(0,1)$, by the continuity of $U$, we then can choose $\alpha \in(0, \epsilon)$ sufficiently small such that

$$
\frac{\sup _{\|x\|_{H} \leq \alpha} U(x)}{c_{2} \epsilon^{2}}<\frac{\epsilon}{2} .
$$

Denote by $X^{\xi}(t)$ and $X^{\eta}(t)$ two different mild solutions to (2.1) starting from initial datums $\xi$ and $\eta$, respectively. Let $\mathcal{K}$ be any compact subset of $H$ and fix any $\xi, \eta \in \mathcal{K}$. For $\beta>\alpha$, we define two stopping times as follows:

$\tau_{\alpha}=\inf \left\{t \geq 0:\left\|X^{\xi}(t)-X^{\eta}(t)\right\|_{H} \leq \alpha\right\}, \tau_{\beta}=\inf \left\{t \geq 0:\left\|X^{\xi}(t)-X^{\eta}(t)\right\|_{H} \geq \beta\right\}$. 
Set $t_{\beta}=\tau_{\beta} \wedge t$. Using the Itô formula (i.e. Lemma 2.2) on the function $U(x)$ and strong solution $X_{n}^{\xi}(t)-X_{n}^{\eta}(t)$ to (3.6), we compute by (3.8)

$$
\begin{aligned}
& \mathbb{E} U\left(X_{n}^{\xi}\left(t_{\beta}\right)-X_{n}^{\eta}\left(t_{\beta}\right)\right) \\
& =\mathbb{E} U(R(n)(\xi-\eta))+\mathbb{E} \int_{0}^{t_{\beta}} \mathcal{L}_{n} U\left(X_{n}^{\xi}(s), X_{n}^{\eta}(s)\right) d s \\
& =\mathbb{E} U(R(n)(\xi-\eta))+\mathbb{E} \int_{0}^{t_{\beta}} \mathcal{L} U\left(X_{n}^{\xi}(s), X_{n}^{\eta}(s)\right) d s \\
& +\mathbb{E} \int_{0}^{t_{\beta}}\left[\mathcal{L}_{n} U\left(X_{n}^{\xi}(s), X_{n}^{\eta}(s)\right)-\mathcal{L} U\left(X_{n}^{\xi}(s), X_{n}^{\eta}(s)\right)\right] d s \\
& \leq \mathbb{E} U(R(n)(\xi-\eta))-\lambda_{2} \mathbb{E} \int_{0}^{t_{\beta}} U\left(X_{n}^{\xi}(s)-X_{n}^{\eta}(s)\right) d s \\
& +\mathbb{E} \int_{0}^{t_{\beta}}(R(n)-I)\left(F\left(X_{n}^{\xi}(s)\right)-F\left(X_{n}^{\xi}(s)\right)\right) d s \\
& +\frac{1}{2} \mathbb{E} \int_{0}^{t_{\beta}} \operatorname{tr}\left[U_{x x}\left(X_{n}^{\xi}(s)-X_{n}^{\eta}(s)\right) R(n)\left(G\left(X_{n}^{\xi}(s)\right)-G\left(X_{n}^{\xi}(s)\right)\right)\right. \\
& \left.\times Q\left(R(n)\left(G\left(X_{n}^{\xi}(s)\right)-G\left(X_{n}^{\xi}(s)\right)\right)\right)^{*}\right] d s \\
& -\frac{1}{2} \mathbb{E} \int_{0}^{t_{\beta}} \operatorname{tr}\left[U_{x x}\left(X_{n}(s)\right)\left(G\left(X_{n}^{\xi}(s)\right)-G\left(X_{n}^{\xi}(s)\right)\right) Q\left(G\left(X_{n}^{\xi}(s)\right)-G\left(X_{n}^{\xi}(s)\right)\right)^{*}\right] d s .
\end{aligned}
$$

Next, taking into account Lemma 2.3 and the dominated convergence theorem yields that

$$
\mathbb{E} U\left(X^{\xi}\left(t_{\beta}\right)-X^{\eta}\left(t_{\beta}\right)\right) \leq \mathbb{E} U(\xi-\eta)-\lambda_{2} \mathbb{E} \int_{0}^{t_{\beta}} U\left(X^{\xi}(s)-X^{\eta}(s)\right) d s .
$$

By (3.7), it directly follows that

$$
c_{2} \mathbb{E}\left[\left\|X^{\xi}\left(\tau_{\beta}\right)-X^{\eta}\left(\tau_{\beta}\right)\right\|_{H}^{2} I_{\left\{\tau_{\beta} \leq t\right\}}\right] \leq \mathbb{E} U(\xi-\eta),
$$

which, together with the definition of $\tau_{\beta}$, gives that

$$
P\left\{\tau_{\beta} \leq t\right\} \leq \frac{\mathbb{E} U(\xi-\eta)}{c_{2} \beta^{2}} .
$$

Hence, there exists a $\beta=\beta(\mathcal{K}, \epsilon)>0$ such that

$$
P\left\{\tau_{\beta}<\infty\right\} \leq \frac{\epsilon}{4} .
$$

Fix the $\beta$ and let $t_{\alpha}=\tau_{\alpha} \wedge \tau_{\beta} \wedge t$. In the same way as (3.11) was done, we can obtain from (3.7) that

$$
\begin{aligned}
\mathbb{E} U\left(X^{\xi}\left(t_{\alpha}\right)-X^{\eta}\left(t_{\alpha}\right)\right) & \leq \mathbb{E} U(\xi-\eta)-\lambda_{2} \mathbb{E} \int_{0}^{t_{\alpha}} U\left(X^{\xi}(s)-X^{\eta}(s)\right) d s \\
& \leq \mathbb{E} U(\xi-\eta)-c_{2} \lambda_{2} \mathbb{E} \int_{0}^{t_{\alpha}}\left\|X^{\xi}(s)-X^{\eta}(s)\right\|_{H}^{2} d s \\
& \leq \mathbb{E} U(\xi-\eta)-c_{2} \lambda_{2} \alpha^{2} \mathbb{E}\left(\tau_{\alpha} \wedge \tau_{\beta} \wedge t\right) .
\end{aligned}
$$

So

$$
P\left\{\tau_{\alpha} \wedge \tau_{\beta} \geq t\right\} \leq \frac{\mathbb{E} U(\xi-\eta)}{c_{2} \lambda_{2} \alpha^{2} t}
$$


which furthermore implies that for given $\epsilon \in(0,1)$ there exists a constant $T=$ $T(\mathcal{K}, \epsilon)>0$ such that

$$
P\left\{\tau_{\alpha} \wedge \tau_{\beta} \leq T\right\}>1-\frac{\epsilon}{4}
$$

By (3.12)

$$
1-\frac{\epsilon}{4}<P\left\{\tau_{\alpha} \wedge \tau_{\beta} \leq T\right\} \leq P\left\{\tau_{\alpha} \leq T\right\}+P\left\{\tau_{\beta}<\infty\right\} \leq P\left\{\tau_{\alpha} \leq T\right\}+\frac{\epsilon}{4},
$$

which yields

$$
P\left\{\tau_{\alpha} \leq T\right\} \geq 1-\frac{\epsilon}{2} .
$$

Now, define the stopping time

$$
\sigma=\inf \left\{t \geq \tau_{\alpha} \wedge T:\left\|X^{\xi}(t)-X^{\eta}(t)\right\|_{H} \geq \epsilon\right\} .
$$

Let $t>T$ and compute

$$
\begin{aligned}
c_{2} \epsilon^{2} P\left\{\tau_{\alpha} \leq T, \sigma \leq t\right\} & \leq \mathbb{E} I_{\left\{\tau_{\alpha} \leq T, \sigma \leq t\right\}} U\left(X^{\xi}(\sigma \wedge t)-X^{\eta}(\sigma \wedge t)\right) \\
& \leq \mathbb{E} I_{\left\{\tau_{\alpha} \leq T\right\}} U\left(X^{\xi}\left(\tau_{\alpha} \wedge t\right)-X^{\eta}\left(\tau_{\alpha} \wedge t\right)\right) \\
& \leq \mathbb{E} I_{\left\{\tau_{\alpha} \leq T\right\}} U\left(X^{\xi}\left(\tau_{\alpha}\right)-X^{\eta}\left(\tau_{\alpha}\right)\right) \\
& \leq P\left\{\tau_{\alpha} \leq T\right\} \sup _{\|x\|_{H} \leq \alpha} U(x) .
\end{aligned}
$$

This, together with (3.10) and (3.14), gives

$$
P\left\{\tau_{\alpha} \leq T, \sigma \leq t\right\}<\frac{\epsilon}{2} .
$$

While, by (3.14) and (3.15)

$$
P\{\sigma \leq t\} \leq P\left\{\tau_{\alpha} \leq T, \sigma \leq t\right\}+P\left\{\tau_{\alpha}>T\right\}<\epsilon .
$$

Letting $t \rightarrow \infty$, we have

$$
P\{\sigma<\infty\} \leq \epsilon .
$$

This implies that for any $\xi, \eta \in \mathcal{K}$, we must have that for $t \geq T$

$$
P\left\{\left\|X^{\xi}(t)-X^{\eta}(t)\right\|_{H}<\epsilon\right\} \geq 1-\epsilon,
$$

as required.

Let $\mathscr{P}(H)$ denote all probability measures on $H$. For $P_{1}, P_{2} \in \mathscr{P}(H)$ define the metric

$$
d_{\mathbb{L}}\left(P_{1}, P_{2}\right)=\sup _{f \in L}\left|\int_{H} f(x) P_{1}(d x)-\int_{H} f(x) P_{2}(d x)\right|
$$

and

$$
\mathbb{L}=\left\{f: H \rightarrow R:|f(x)-f(y)| \leq\|x-y\|_{H} \text { and }|f(\cdot)| \leq 1\right\} .
$$

Lemma 3.3. Let (3.9) hold. Then, for any compact subset $\mathcal{K}$ of $H$,

$$
\lim _{t \rightarrow \infty} d_{\mathbb{L}}(p(t, \xi, \cdot), p(t, \zeta, \cdot))=0, \quad \text { uniformly in } \xi, \zeta \in \mathcal{K} \text {. }
$$


Proof. It is sufficient to show that there is a $\bar{T}>0$ such that for any $\varepsilon>0$ and $\xi, \zeta \in \mathcal{K}$

$$
d_{\mathbb{L}}(p(t, \xi, \cdot), p(t, \zeta, \cdot)) \leq \varepsilon, \quad \forall t \geq \bar{T},
$$

which is equivalent to showing

$$
\sup _{f \in \mathbb{L}}\left|\mathbb{E} f\left(X^{\xi}(t)\right)-\mathbb{E} f\left(X^{\zeta}(t)\right)\right| \leq \varepsilon, \quad \forall t \geq \bar{T} .
$$

However, for any $f \in \mathbb{L}$,

$$
\left|\mathbb{E} f\left(X^{\xi}(t)\right)-\mathbb{E} f\left(X^{\zeta}(t)\right)\right| \leq \mathbb{E}\left(2 \wedge\left\|X^{\xi}(t)-X^{\zeta}(t)\right\|_{H}\right) .
$$

By (3.9) there exists a $\bar{T}>0$ satisfying

$$
\mathbb{E}\left(2 \wedge\left\|X^{\xi}(t)-X^{\zeta}(t)\right\|_{H}\right) \leq \varepsilon, \quad \forall t \geq \bar{T} .
$$

Since $f \in \mathbb{L}$ is arbitrary, we have that

$$
\sup _{f \in \mathbb{L}}\left|\mathbb{E} f\left(X^{\xi}(t)\right)-\mathbb{E} f\left(X^{\zeta}(t)\right)\right| \leq \varepsilon, \quad \forall t \geq \bar{T} .
$$

The desired result then follows.

Lemma 3.4. Let (3.3) and (3.9) hold. Then, $\{p(t, \xi, \cdot): t \geq 0\}$ is Cauchy in the space $\mathscr{P}(H)$ for any $\xi \in H$.

Proof. Fix $\xi \in H$. We need to show that for any $\varepsilon>0$ there exists a $T>0$ such that

$$
d_{\mathbb{L}}(p(t+s, \xi, \cdot), p(t, \xi, \cdot)) \leq \varepsilon, \quad \forall t \geq T, s>0,
$$

which is equivalent to showing that

$$
\sup _{f \in \mathbb{L}}\left|\mathbb{E} f\left(X^{\xi}(t+s)\right)-\mathbb{E} f\left(X^{\xi}(t)\right)\right| \leq \varepsilon, \quad \forall t \geq T, s>0 .
$$

From Remark 3.1 we know that there exists a compact subset $\mathcal{K}$ of $H$ such that for any $\varepsilon>0$

$$
p(s, \xi, \mathcal{K})>1-\frac{\varepsilon}{8} .
$$

Next, by the strong Markov property of $X^{\xi}(t)$, for any $f \in \mathbb{L}$ and $t, s>0$

$$
\begin{aligned}
\left|\mathbb{E} f\left(X^{\xi}(t+s)\right)-\mathbb{E} f\left(X^{\xi}(t)\right)\right| & =\left|\mathbb{E}\left[\mathbb{E} f\left(X^{\xi}(t+s)\right) \mid \mathcal{F}_{s}\right]-\mathbb{E} f\left(X^{\xi}(t)\right)\right| \\
& =\left|\int_{H} \mathbb{E} f\left(X^{\zeta}(t)\right) p(s, \xi, d \zeta)-\mathbb{E} f\left(X^{\xi}(t)\right)\right| \\
& \leq \int_{H} \mathbb{E}\left|f\left(X^{\zeta}(t)\right)-f\left(X^{\xi}(t)\right)\right| p(s, \xi, d \zeta) \\
& =\int_{\mathcal{K}} \mathbb{E}\left|f\left(X^{\zeta}(t)\right)-f\left(X^{\xi}(t)\right)\right| p(s, \xi, d \zeta) \\
& +\int_{H-\mathcal{K}} \mathbb{E}\left|f\left(X^{\zeta}(t)\right)-f\left(X^{\xi}(t)\right)\right| p(s, \xi, d \zeta) \\
& \leq \int_{\mathcal{K}} \mathbb{E}\left|f\left(X^{\zeta}(t)\right)-f\left(X^{\xi}(t)\right)\right| p(s, \xi, d \zeta)+\frac{\varepsilon}{4},
\end{aligned}
$$

where (3.18) has been used in the last inequality. By (3.9) there is a $T>0$ such that for the given $\varepsilon>0$

$$
\mathbb{E}\left|f\left(X^{\zeta}(t)\right)-f\left(X^{\xi}(t)\right)\right| \leq \mathbb{E}\left(2 \wedge\left\|X^{\xi}(t)-X^{\zeta}(t)\right\|_{H}\right) \leq \frac{3 \varepsilon}{4}, \quad \forall t \geq T .
$$


Hence, in (3.19)

$$
\left|\mathbb{E} f\left(X^{\xi}(t+s)\right)-\mathbb{E} f\left(X^{\xi}(t)\right)\right| \leq \varepsilon, \quad \forall t \geq T, s>0 .
$$

Since $f \in \mathbb{L}$ is arbitrary, the desired inequality (3.17) follows.

After the preparation of the lemmas above, we now can state our main result.

Theorem 3.1. Under the conditions of Lemma 3.1 and Lemma 3.2, the mild solution $X(t)$ to (2.1) is stable in distribution.

Proof. By the definition of stability in distribution of mild solutions, it is sufficient to show that there exists a probability measure $\pi(\cdot)$ such that for any $\xi \in H$, the transition probabilities $\{p(t, \xi, \cdot): t \geq 0\}$ converge weakly to $\pi(\cdot)$. As we know, the weak convergence of probability measures is equivalent to a metric concept; therefore we need to show that for any $\xi \in H$,

$$
\lim _{t \rightarrow \infty} d_{\mathbb{L}}(p(t, \xi, \cdot), \pi(\cdot))=0 .
$$

By Lemma 3.4 $\{p(t, 0, \cdot): t \geq 0\}$ is Cauchy in the space $\mathscr{P}(H)$ with metric $d_{\mathbb{L}}$. Since $\mathscr{P}(H)$ is a complete metric space under metric $d_{\mathbb{L}}$ (cf. [4, Theorem 5.4]), there is a unique probability measure $\pi(\cdot) \in \mathscr{P}(H)$ such that

$$
\lim _{t \rightarrow \infty} d_{\mathbb{L}}(p(t, 0, \cdot), \pi(\cdot))=0 .
$$

Moreover,

$$
\lim _{t \rightarrow \infty} d_{\mathbb{L}}(p(t, \xi, \cdot), \pi(\cdot)) \leq \lim _{t \rightarrow \infty} d_{\mathbb{L}}(p(t, \xi, \cdot), p(t, 0, \cdot))+\lim _{t \rightarrow \infty} d_{\mathbb{L}}(p(t, 0, \cdot), \pi(\cdot)) .
$$

This, together with (3.20), means

$$
\lim _{t \rightarrow \infty} d_{\mathbb{L}}(p(t, \xi, \cdot), \pi(\cdot))=0 .
$$

\section{EXAMPLE}

In this section, in order to demonstrate the applicability of our theory, one example is constructed.

Example 4.1. Consider the semi-linear stochastic partial differential equation

$$
\left\{\begin{array}{l}
d y(x, t)=\frac{\partial^{2}}{\partial x^{2}} y(x, t) d t+\sigma f(y(x, t)) d W(t), \quad t \geq 0,0<x<1 \\
y(0, t)=y(1, t)=0, \quad t \geq 0 ; \quad y(x, 0)=y_{0}(x), \quad 0 \leq x \leq 1
\end{array}\right.
$$

where $W(t), t \geq 0$, is a real standard Brownian motion, $\sigma$ is a real number and $f$ is a real Lipschitz continuous function on $L^{2}(0,1)$ satisfying for $u, v \in L^{2}(0,1)$ and some positive constants $c, k$,

$$
\begin{aligned}
|f(u)| & \leq c\left(\|u\|_{H}+1\right), \\
|f(u)-f(v)| & \leq k\|u-v\|_{H} .
\end{aligned}
$$

In this example, we take $H=L^{2}(0,1)$ and $A=\frac{\partial^{2}}{\partial x^{2}}$ with $\mathcal{D}(A)=H_{0}^{1}(0,1) \cap$ $H^{2}(0,1)$. Then, by Govindan [8], for any $u \in \mathcal{D}(A)$,

$$
\langle u, A u\rangle_{H} \leq-\pi^{2}\|u\|_{H}^{2} .
$$

For all $u \in \mathcal{D}(A)$

$$
\mathcal{L}\|u\|_{H}^{2}=2\langle u, A u\rangle_{H}+\sigma^{2}|f(u)|^{2} \leq-2\left(\pi^{2}-\sigma^{2} c^{2}\right)\|u\|_{H}^{2}+2 \sigma^{2} c^{2} .
$$


Similarly,

$$
\mathcal{L}\|u-v\|_{H}^{2} \leq-\left(2 \pi^{2}-\sigma^{2} k^{2}\right)\|u-v\|_{H}^{2}
$$

Therefore, if $\sigma^{2} c^{2}<\pi^{2}$ and $\sigma^{2} k^{2}<2 \pi^{2}$, then we immediately deduce by Theorem 3.1 that the mild solution process $y(x, t)$ of (4.1) is stable in distribution.

\section{REFERENCES}

[1] G.K. Basak, A. Bisi and M.K. Ghosh, Stability of a random diffusion with linear drift, J. Math. Anal. Appl., 202 (1996), 604-622. MR1406250(97g:60091)

[2] T. Caraballo and J. Real, On the pathwise exponential stability of non-linear stochastic partial differential equations, Stoch. Anal. Appl., 12 (1994), 517-525. MR.1297111 (95k:60147)

[3] T. Caraballo and K. Liu, On exponential stability criteria of stochastic partial differential equations, Stoch. Proc. Appl., 83 (1999), 289-301. MR1708210 (2000h:60067)

[4] M.F. Chen, From Markov Chains to Non-equilibrium Particle Systems, World Scientific, Singapore, 1992. MR2091955 (2005h:60004)

[5] P.L. Chow, Stability of nonlinear stochastic-evolution equations, J. Math. Anal. Appl., 89 (1982), 400-419. MR677738(84h:60111)

[6] G. Da Prato and J. Zabczyk, Stochastic Equations in Infinite Dimensions, Cambridge University Press, 1992. MR:1207136 (95g:60073)

[7] G. Da Prato and J. Zabczyk, Ergodicity for Infinite-Dimensional Systems, Cambridge University Press, 1996. MR:1417491 (97k:60165)

[8] T.E. Govindan, Almost sure exponential stability for stochastic neutral partial functional differential equations, Stochastics, 77 (2005), 139-154. MR2151664 (2006d:60103)

[9] U.G. Haussmann, Asymptotic stability of the linear Itô equation in infinite dimensions, J. Math. Anal. Appl., 65 (1978), 219-235. MR501750 (80b:60082)

[10] A. Ichikawa, Stability of semilinear stochastic evolution equations, J. Math. Anal. Appl., 90 (1982), 12-44. MR680861 (84g:60091)

[11] A. Ichikawa, Absolute stability of a stochastic evolution equation, Stochastics, 11 (1983), 143-158. MR729494 (87a:60067)

[12] R. Liu and V. Mandrekar, Stochastic semilinear evolution equations: Lyapunov function, stability, and ultimate boundedness, J. Math. Anal. Appl., 212 (1997), 537-553. MR 1464896 (98k:60108)

[13] T. Taniguchi, The exponential stability for stochastic delay partial differential equations, J. Math. Anal. Appl., 331 (2007), 191-205. MR2305998(2008e:60191)

[14] C. Yuan and X. Mao, Asymptotic stability in distribution of stochastic differential equations with Markovian switching, Stoch. Proc. Appl., 103 (2003), 277-291. MR.1950767 (2003k:60142)

School of Mathematics, Central South University, Changsha, Hunan 410075, People's Republic of China

School of Mathematics, Central South University, Changsha, Hunan 410075, People's Republic of China

Department of Mathematics, Swansea University, Swansea SA2 8PP, United Kingdom

E-mail address: C.Yuan@Swansea.ac.uk 